\title{
Application of image enhancement and mixture of Gaussian approach in combustion research
}

\author{
LITU ROUT ${ }^{1}$, RAJESH SADANANDAN $^{2}$ and DEEPAK MISHRA ${ }^{1, *_{0}}$ \\ ${ }^{1}$ Department of Avionics, Indian Institute of Space Science and Technology, Thiruvananthapuram, \\ Kerala 695 547, India \\ ${ }^{2}$ Department of Aerospace, Indian Institute of Space Science and Technology, Thiruvananthapuram, \\ Kerala 695 547, India \\ e-mail: liturout1997@gmail.com; rajeshsadanandan@iist.ac.in; deepak.mishra@iist.ac.in
}

MS received 10 September 2018; revised 19 February 2019; accepted 21 February 2019; published online 13 April 2019

\begin{abstract}
Chemiluminescence is one of the most commonly used optical diagnostic techniques in combustion research where a line-of-sight projected information is generated from spatial fields. The exactness and uniqueness of reconstruction along with ease of implementation gives Abel inversion an edge over the other existing single-view reconstruction techniques for efficient estimation of spatial field from line-of-sight projections. Though there exist many such algorithms, the primary focus of these has been to ensure tractable inversion through a systematic regularization by imposing a smoothness constraint on discrete data points. But these techniques do not have the provision to process the input image prior to deconvolution in order to prevent accumulation of noise infiltrated during data acquisition. Another major limitation of these algorithms is to adopt the changes in characteristics of the input data points while maintaining optimal storage and time complexity. To address these issues, we have proposed a new image processing technique using standard Abel inversion for the application in combustion research. It provides a suitable model to ensure regularized inversion by imposing a smoothness constraint on acquired raw data. The new algorithm has been implemented to yield the physically significant chemiluminescence emission from hydroxyl radicals in flames from line-of-sight integrated images. The effectiveness of this algorithm is highlighted using exemplary $\mathrm{OH}$ chemiluminescence images captured from a standard swirl stabilized research burner.
\end{abstract}

Keywords. Abel deconvolution; image enhancement; mixture of Gaussian; swirl combustion; optical diagnostics; chemiluminescence.

\section{Introduction}

In this study, we develop an image processing algorithm using Abel inversion for application in combustion research, especially in 2D analysis of chemiluminescence images from flames. The chemical reactions in flames generate energy and the electronic excitation of molecules takes place. The excited molecules undergo deactivation to lower energy levels by the emission of photon, and the process is termed as chemiluminescence. The primary source of chemiluminescence in hydrocarbon flames constitute the radiative emissions from electronically excited species that are formed chemically, such as $\mathrm{CH}^{*}, \mathrm{OH}^{*}, \mathrm{C}_{2}$ * and $\mathrm{CO}_{2} *$ [1]. Under fuel lean conditions chemiluminescence can, therefore, be taken as an indicator of the reacting conditions in the flame since these species are mainly produced in the reaction zone [2] [3]. Of these, the emissions in the UV range stem predominantly from $\mathrm{OH}$

*For correspondence (hydroxyl radical) in an electronically excited state (termed $\left.\mathrm{OH}^{*}\right)$. In combustion research $\mathrm{OH}^{*}$ is, therefore, one of the most commonly used radicals to identify the spatial dimensions of the flame and the heat release behavior. $\mathrm{OH}^{*}$ has a very short lifetime i.e. on the order of a few nanoseconds. From investigations in flames with gaseous fuels, there are indications that the intensity of the $\mathrm{OH}^{*}$ chemiluminescence is related to the reaction rate or heat release rate. So by capturing the $\mathrm{OH}^{*}$ chemiluminescence emissions from the flame (using an intensified CCD camera), information can be obtained about the instantaneous size and shape of heat release zone. Since the measurement technique is line of sight, with the $\mathrm{OH}^{*}$ emissions collected along the line of sight, the spatial resolution is compromised. However, in case of flames from axisymmetric burners, Abel deconvolution can be used to reconstruct the underlying 2D scalar fields or radial distributions. The 2D information so obtained are also vital for the modeling, validation and optimization of numerical simulation codes. 
Various Abel deconvolution algorithms have been developed over the years with diverse applications such as heat conduction, infrared tomography and many more [4-9]. But most of these algorithms focus primarily on developing a strategy to enhance degree of smoothness with minimal consideration given to pre-processing the acquired raw results. In this paper, we propose to implement Abel deconvolution under noisy environment by taking utmost care while acquiring the results (raw images), modeling the noise and implementing a Mixture Of Gaussian (MOG) model to ensure higher degree of smoothness. An extensive investigation has been conducted in deciding the suitable curve that is preferred over the others while fitting the discrete data points. Based on this, a new way of implementing Abel deconvolution, which is robust to noisy and discrete experimental data, has been proposed. The following sections give a brief description about the mathematical aspects and geometrical interpretation (section 2), related works (section 3), followed by the implementation of the algorithm (section 4 ) in a $2 \mathrm{D}^{-\mathrm{OH}^{*}}$ chemiluminescence image acquired from a standard swirl stabilized burner.

\section{A brief review of Abel transform}

In this section 2, a very brief discussion about the forward (2.1) Abel Transform and Abel Deconvolution (2.2) is given. More details on Abel Transform can be found at [10].

\subsection{Forward Abel transform}

The forward Abel transform of a function $f(r)$ is defined by the following equation as

$$
F(y)=2 \int_{y}^{\infty} \frac{f(r) r d(r)}{\sqrt{r^{2}-y^{2}}},
$$

where $r=\sqrt{\left(x^{2}+y^{2}\right)}$ represents the radial distance of the points on the line of sight of integration and $y$ represents the distance of the observer from $\mathrm{X}$ axis as shown in figure 1 [10].

\subsection{Abel deconvolution}

The Abel deconvolution is usually used to obtain the symmetric function from it's projection i.e. a scan or a photograph [10]. Assuming that $f(r), f^{\prime}(r)$ ( $\%$ ' represents first derivative) drops to zeros more quickly than $\frac{1}{r}$, the inverse Abel transform is given by Eq. (2). The emission function $f(r)$ can be obtained from its projection $\mathrm{F}(\mathrm{y})$ with the help of this inverse Abel transform.

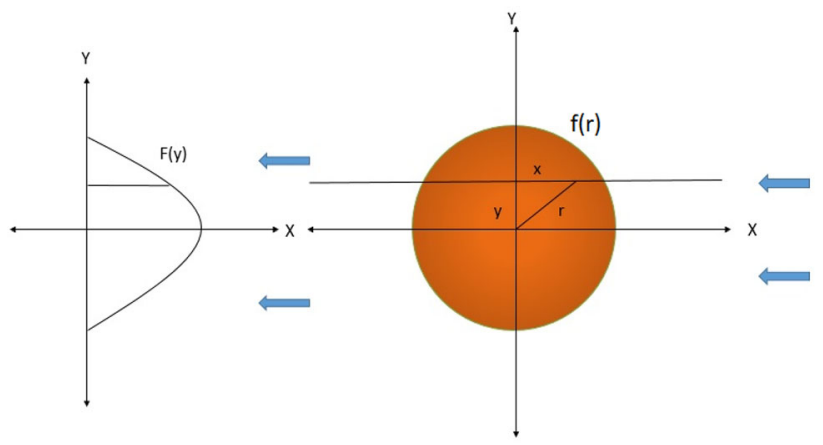

Figure 1. Projection of a transparent, emitting scalar field with circular symmetry as per the field of view of an observer along the blue arrows.

$$
f(r)=\frac{-1}{\pi} \int_{r}^{\infty} \frac{\mathrm{d} F}{\mathrm{~d} y} \frac{d y}{\sqrt{y^{2}-r^{2}}}
$$

For the ease of understanding, we have demonstrated the physical interpretations of Abel transformation with mathematical equations in light of circular symmetry. However, the general equations (1) and (2) of Abel transformation with certain modifications can be extended to other instances, such as spherical and cylindrical symmetry, without the loss of generality.

Of particular interest, the projection of a cylindrical symmetric function $f(\rho, z)$ onto a plane parallel to $\mathrm{Z}$ axis can be obtained by taking Abel transform of $f(\rho, z)$ as shown in the following equation.

$$
F(y, z)=\int_{-\infty}^{\infty} f(\rho, z) d x=2 \int_{y}^{\infty} \frac{f(\rho, z) \rho d \rho}{\sqrt{\rho^{2}-y^{2}}}
$$

where $\rho^{2}=x^{2}+y^{2}$ is the cylindrical radius and $F(y, z)$ is the projection on $\mathrm{YZ}$ plane. The inverse Abel transform of such projection can be obtained by the following equation.

$$
f(\rho, z)=\frac{-1}{\pi} \int_{\rho}^{\infty} \frac{d F}{d y} \frac{d y}{\sqrt{y^{2}-\rho^{2}}}
$$

Since our application provides the projected image $F(y, z)$, we aim to employ inverse Abel transform, as given in Eq. (4), to obtain the cylindrical symmetric flame $f(\rho, z)$.

\section{Related works}

Abel transform and its inverse integral equation finds numerous applications in various branches of science especially in plasma diagnostics and X-ray radiography, where it is used to yield information about the density or temperature distribution [4]. In combustion studies, the deconvolution algorithms are used for extracting the tomography of spectroscopic emissions from axisymmetric objects, for example flames in axisymmetric burners. One 
of the most commonly used algorithms in this case is the onion peeling method [5]. The ill posed sets of equations generated by the onion-peeling scheme are transformed into well posed sets with the help of Tikhonov regularization scheme [6]. A comparative study of Abel three-point deconvolution, onion-peeling and Tikhonov regularization is given [8] for a known spatial field from a line-of-sight integrated projection data.

Other approaches to solving this singular integral equation obtained from axisymmetric flame and parallel scanning measurements are generalized quadrature method, spline smoothing and Tikhonov regularization along with infrared tomography [9].

For an axisymmetric object the measured intensity, in gray level units, is related to the object's radial optical density profile through Abel's integral equation. So, in order to extract the physically significant radial density profile the measured raw data (or image) needs to be Abel inverted, which can then be used for non-destructive evaluation of the object. In other words, the forward Abel transform is used for the projection of an axially symmetric function onto a plane, whereas inverse Abel transform is used for extracting the axially symmetric function from its projection i.e. a scan or a photograph. Computational issues arise when the raw data contains noise because the efficacy of inversion algorithm greatly depends on the suppression of noise. Since the expression used for inversion is an integral equation, it is certainly affected by the accumulation of small drifts due to noise. Also, the derivative used in Abel integral equation tends to amplify the errors. Over the years, many Abel inversion techniques have been proposed $[9,11-13]$. One of the successful algorithms was proposed by Deutsch and Beniaminy [11], that uses derivative free implementation of Abel inversion. One of the key features incorporated in this derivative free inversion is based on the least square smoothing of discrete experimental data. Though the algorithm produces high quality images after inversion, its time complexity is $O\left(n^{1.5}\right)$ and also it requires high storage space. In spline based representation of the input data, the implementation of spline fit avoids amplification of noisy data. But the quality of this fitting procedure deteriorates at the end points of the sampling interval. Therefore, a plausible solution to address this issue would be to reflect part of the data relative to $y$-axis before fitting the data using a spline fit [13].

The analytic spline Abel inversion (ASAI) is another successful implementation of Abel deconvolution that has been proved to be extremely effective under noisy and noise free environment. Despite the effectiveness of ASAI algorithm, it requires $O\left(n^{2}\right)$ number of arithmetic operations that includes four transcendental operations per each set of data and also, its coefficients are uniquely associated with the data points. Hence these coefficients can't be stored in advance. In order to address this issue Gueron and Deutsch have proposed a fast Abel inversion (FAI) technique [13] that uses less number of transcendental operations while using the spline based smoothing procedure. The FAI algorithm computes coefficients which are independent of the data points and hence, these can be computed once, and used for many dataset [13]. Though this independent approach is considered to be significantly impressive, there is a need to change the coefficients slightly in order to adapt to the changes in data points. Therefore, a smoothing scheme which can adapt to the changes with less number of arithmetic operations, especially transcendental operations, and a high degree of smoothness is desirable.

Though the smoothing procedure eliminates the abrupt transition in intensities, we have observed that noise due to various sources still persists in the captured images. Therefore, in this paper, we have experimented various standard image enhancement schemes to further alleviate the adverse effects of image acquisition in real world applications.

\section{Proposed methodology}

In the proposed algorithm we assume that the line-of-sight projections of the spatial field are equally spaced over the whole field and each of these projections are obtained with the help of a narrow beam. It has been proved that sampling has a great influence on the quantity of noise which gets added to the spatial field during the process of deconvolution [5].

Therefore one of the most important steps in deconvolution is to eliminate oversampling and smooth the projected discrete data prior to deconvolution. While the oversampling issue is resolved by taking utmost care during image acquisition, the Mixture of Gaussian (MOG) model, as proposed in this paper, has been used to approximate the discrete noisy experimental data.

In the following sections, we elaborate on the proposed methodology with detailed experimentation and analysis. The image acquiring process and image enhancement (section 4.1) techniques have been discussed prior to estimation of smoothing function (section 4.2) and Abel inversion (section 4.3). Thereafter, the post-processing task such as spatial distribution of deconvoluted data points has been discussed (section 4.4), followed by the essential algorithms (section 4.5) namely coefficient extraction and Abel deconvolution.

\subsection{Image acquisition and enhancement}

Space and time complexities are two of the important factors in most image processing techniques. Storage and retrieval without loss of valuable information is nonetheless challenging task in various scenarios. In order to deal with such problems, we have taken utmost care during data 
acquisition. The $\mathrm{OH}^{*}$ emission images from a standard swirl burner, as used in this work, is captured using an intensified CCD camera. The raw images are recorded in .im7 format (type: graphic) which is a proprietary file format from LaVision GmbH. For the post processing of the images the .im7 images are exported into .jpeg format. After storing the data, the next vital step is to remove noise using image enhancement techniques as a regularization strategy to make Abel inversion more tractable.

In real world applications, it is almost intractable to acquire images without noise. In image processing perspective, it can be justified by central limit theorem that these unwanted signals, often referred to as noise, tend to follow Gaussian distribution when several random numbers are added [14]. Thus, the unavoidable noise, since it affects all the pixels of the captured image, is reasonable to be modelled as Additive White Gaussian Noise (AWGN). Hence, an edge preserved smoothing mechanism will be a great option to eliminate the AWGN without corrupting the data. However, it is extremely difficult to compute the latent variables of such distribution and eliminate the noise completely. Therefore, one of the plausible solutions to tackle such an issue is to employ various image enhancement techniques on the raw pixels and analyze the impact of such methods qualitatively. Since neither the noise-free image nor the actual noise distribution is at hand, we have preferred qualitative analysis over quantitative measures such as SNR. In order to validate our assumption of AWGN, we have experimented with variance and logarithmic transform based enhancement techniques. After exploring these methods, we have observed that edge adaptive smoothing using Gaussian kernels turns out to be more effective when compared with other image enhancement algorithms such as unsharp masking and retinex. Therefore, this comparison validates the additive property of the noise in our raw data up to some extent. One can find a detailed description with notable comparisons among these image enhancement techniques in [14]. The qualitative analysis of the aforementioned techniques is shown in figure 2. As figure 2(b) depicts, the edge preserved smoothing using Gaussian kernel outperforms the rest mechanisms, and hence, it has opted for further processing. In the following few paragraphs, we briefly discuss some key enhancement techniques that we have experimented in this study.

In edge preserved Gaussian smoothing, a small window Waround a pixel is taken. The variance of all the possible windows $w$ of the same dimension in the local neighbourhood that consists of at least one pixel from the window $W$ is computed. Then the central pixel of $W$ is replaced with a smoothed value of the window $w$ that has minimum variance. For a $5 \times 5$ window $W$, there are 25 possible local windows out of which 1 window $w$ has a minimum variance which is used for computing the value of the center pixel [14].

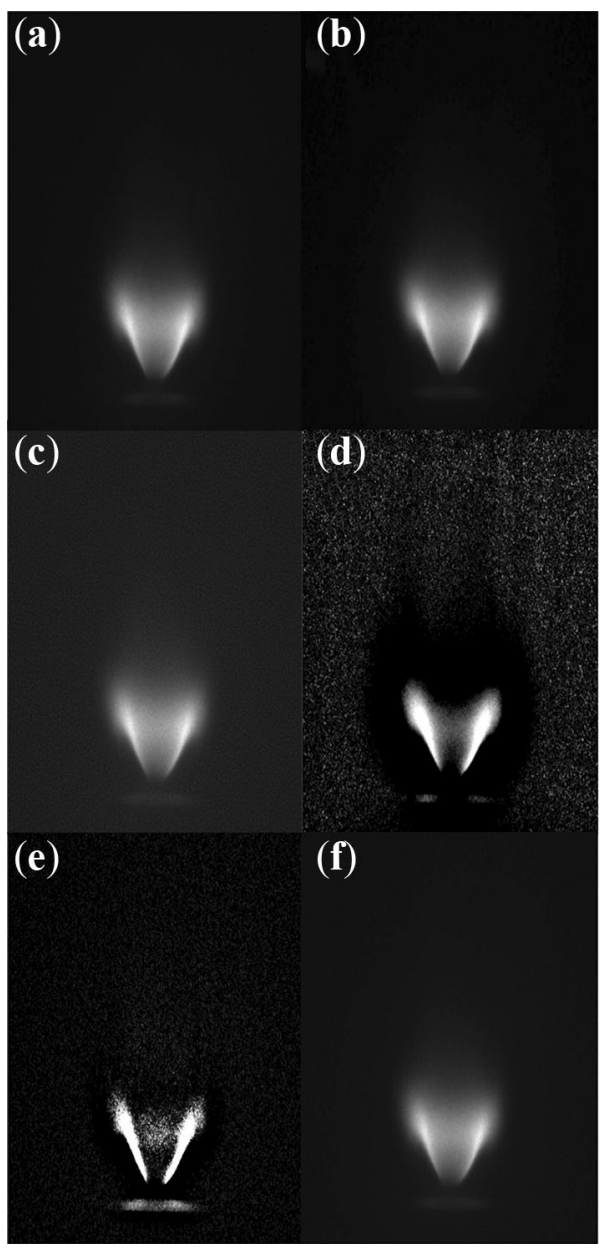

Figure 2. Qualitative analysis of various image enhancement techniques. (a) Noisy discrete experimental data. (b) Edge preserved Gaussian smoothing. (c) Unsharp masking using adaptive Gaussian window. (d) Retinex algorithm with 10 iteration. (e) Unsharp masking using global window. (f) Image sharpening.

In local unsharp masking a local window whereas in global unsharp masking a global window is considered while computing a low pass filtered image. The low pass filtered version of the image is then subtracted from itself and multiplied by an amplification factor. The difference is amplified only when it exceeds a certain threshold which is used to suppress small high frequency fluctuations due to noise. Thus, the amplification factor is chosen according to the local variance. This transformation can be achieved using the following equation [14].

$$
F(y, z)=A[F(y, z)-m(y, z)]+m(y, z)
$$

where $A=\frac{k M}{\sigma(y, z)}, k$ is scalar, $M$ is the average grey value of the whole image, $\sigma(y, z)$ variance of the local window, $m(y, z)$ is the low pass version of $F(y, z)$ and $F(y, z)$ is the line-of-sight integrated image with $y$ and $z$ representing columns and rows respectively. 
The results are compared with single scale retinex or logarithmic transform which is usually used to isolate unwanted signals due to variable illumination that may manifest during image acquisition. It has two basic ingredients:

1. grey value normalization by dividing local mean value;

2. conversion into a logarithmic scale which will enhance dark pixels more and bright pixels less.

This transformation can be achieved by using the following equation.

$$
F(y, z)=\ln (F(y, z)+1)-\ln (\overline{F(y, z)})
$$

where $\overline{F(y, z)}$ is the local mean value of $F(y, z)$. The mean value is obtained using a large Gaussian filter. A deep insight into all these image enhancement techniques can be found in [14]. Here, we essentially employ AWGN filter to enhance the required details of the captured image due to its close approximation to eliminate noise to some degree.

\subsection{Function estimation}

After removing the unwanted signals from the acquired image, the discrete data points are approximated by a continuous function. Here, we discuss various approximating functions and their corresponding goodness of fit (GOF). In order to select the right fit for the discrete experimental data, we have experimented various functions including polynomial, Gaussian, Fourier, smoothing spline, the sum of sine, exponential, power, rational, and Weibull. Based on their performance and required criteria, the feasible functions have been narrowed down to the top three approximators which are mixture of Gaussian (MOG), Fourier series expansion, and higher order polynomial. Also, we have observed that there is an insignificant change in the accuracy of approximation as the order becomes higher and higher. However, the computational cost grows significantly as the order increases. So, the order of the approximating function (here, 8) has been decided such that it provides an optimal residual sum of errors with minimal computational complexity.

In our notation, $F(y, z)$ represents the intensity at $(y, z)$ location of input image in spatial domain. We aim to approximate each row $(z)$ of $F(y, z)$ with appropriate continuous functions and perform Abel inversion to obtain elements of $f(\rho, z)$ in that particular row. The proposed mixture of Gaussian distributions, which is used to approximate discrete $F(y, z)$, is given by the following equation.

$$
F(y, z)=\sum_{n=1}^{8}\left(a_{n} \exp ^{-\left(\frac{y-b_{n}}{c_{n}}\right)^{2}}\right), \forall z
$$

where $a_{n}$ is the maximum amplitude, $b_{n}$ is the mean position and $c_{n}$ is $\sqrt{2}$ times standard deviation $(\sigma)$. Similarly, the
Fourier series approximation is given by the following equation.

$$
F(y, z)=a_{0}+\sum_{n=1}^{8}\left(a_{n} \cos (n \omega)+b_{n} \sin (n \omega)\right), \forall z
$$

where $\omega$ is the fundamental frequency, $a_{n}$ and $b_{n}$ are the coefficients of cosines and sines of $n^{\text {th }}$ harmonic respectively, $a_{0}$ is the offset value. The polynomial model is given by the following equation.

$$
F(y, z)=\sum_{n=1}^{8}\left(a_{n} y^{n}\right), \forall z
$$

where $a_{n}$ represents the coefficients of the polynomial interpolation. Here, the coefficients used in these models are within $95 \%$ confidence bounds [15-17]. A line-of-sight integrated image is shown in figure 3. Polynomial, Gaussian, and Fourier approximation for a fixed row(here, 368 from top) of the line-of-sight integrated image is illustrated in figure 4. As described above, a proper goodness of fit decides the accuracy of the spatial field. Therefore, the Gaussian mixture model has been selected for further processing, as it is found to outperform the rest with minimal error, high goodness of fit and $95 \%$ confidence bound in the region of interest i.e. from column 100 to 200. For better understanding, we have visualized the individual Gaussian distributions along with MOG model in figure 5.

A comparative study of GOF of top performing function approximators has been detailed in Table 1. We have used Regression Squared(R-squared), Adjusted R-squared(AdjR-squared) and Root Mean Squared Error(RMSE) as the essential statistical measures for assessing the quality of fit. The details of these metric can found at [18].

The graphical interpretation (figure 4), as well as quantitative measure (Table 1) clearly indicates that the mixture of Gaussian model has exceedingly high goodness of fit in the region of interest. Outside this region there are dark

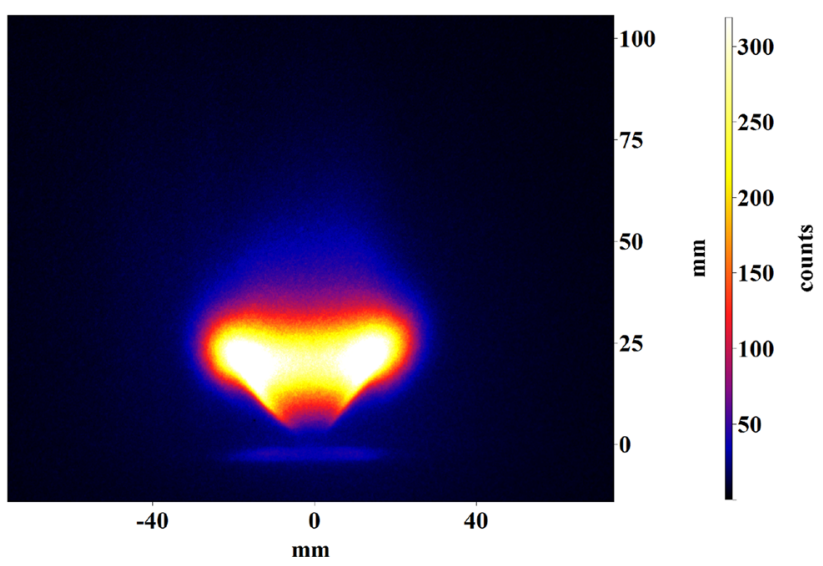

Figure 3. An example of a line-of-sight integrated image. 


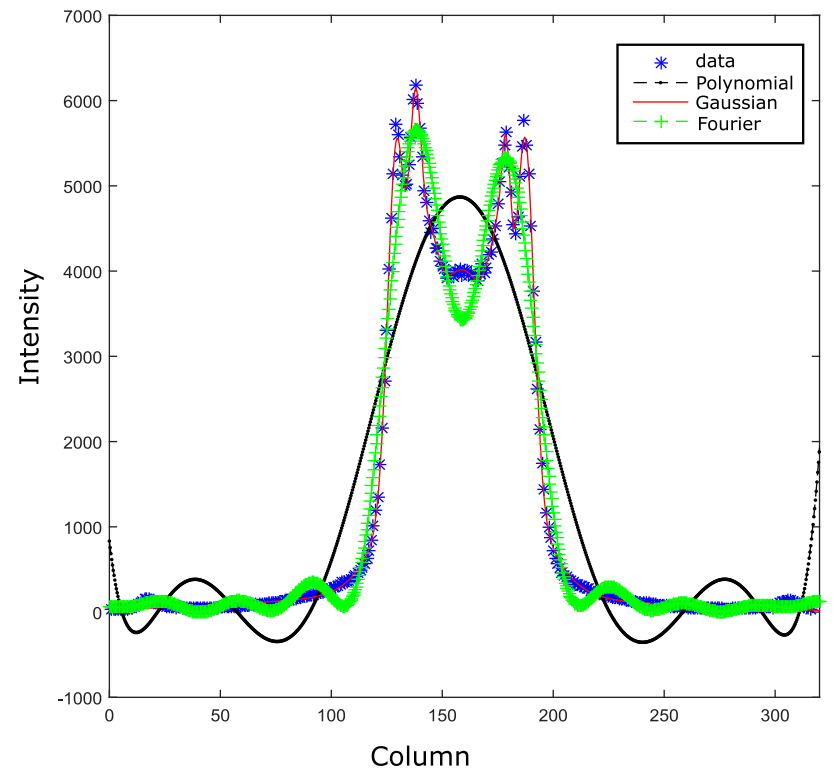

Figure 4. Comparison among Polynomial, Gaussian and Fourier approximation of discrete data points. It is observed that the Gaussian mixture model approximates the data significantly better than Polynomial and Fourier approximation.

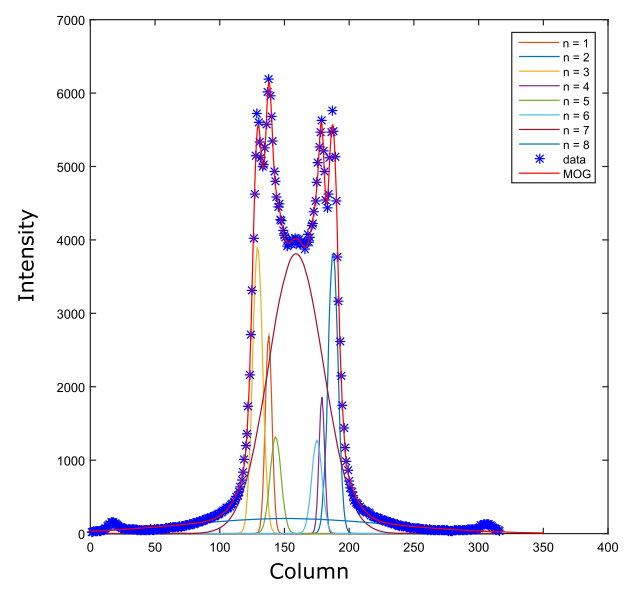

Figure 5. Mixture of eight individual Gaussian distributions.

Table 1. Statistical inference of Goodness Of Fit (GOF).

\begin{tabular}{lccc}
\hline Statistical inference & Polynomial & Fourier & MOG \\
\hline Regression squared (R-squared) & 0.8544 & 0.9750 & 0.9994 \\
$\begin{array}{l}\text { Adjusted R-squared (AdjR- } \\
\quad \text { squared) }\end{array}$ & 0.8506 & 0.9736 & 0.9993 \\
$\begin{array}{l}\text { Root Mean Square Error (RMSE) } \\
722.7\end{array}$ & 303.6 & 47.9 \\
\hline
\end{tabular}

patches of background information which is not of much importance in such applications. The underlying hypothesis of function approximation is that an unknown distribution can be well approximated by mixture of Gaussian when sufficiently large number of Gaussian basis functions are considered. This hypothesis is supported by the central limit theorem and is well investigated in many signal processing applications including image enhancement and interpolation [14]. On the other hand, the polynomial and Fourier series approximation does not fit the data as efficiently as MOG with limited number of free parameters. Therefore, we have opted the MOG model for estimating the continuous approximation of discrete data. The parameters of MOG model i.e. $a_{n}, b_{n}$ and $c_{n}$ are computed by optimizing the squared sum of errors. In this process, the parameters are initialized with random values and then successively iterated until convergence within a prescribed limit. The partial derivatives used to compute the increments are given by

$$
\begin{aligned}
& \frac{\partial F}{\partial a_{1}}=e^{-\left(\frac{y-b_{1}}{c 1}\right)^{2}}, \\
& \frac{\partial F}{\partial b_{1}}=2 a_{1} \frac{\left(y-b_{1}\right)}{c_{1}^{2}} e^{-\left(\frac{y-b_{1}}{c 1}\right)^{2}} \text { and } \\
& \frac{\partial F}{\partial c_{1}}=2 a_{1} \frac{\left(y-b_{1}\right)^{2}}{c_{1}^{3}} e^{-\left(\frac{y-b_{1}}{c 1}\right)^{2}} .
\end{aligned}
$$

This technique could obviously be generalized to multiple Gaussian basis functions, although the convergence property gets worse as the number of parameters increases. However, it is necessary, in some scenarios, to increase the number of Gaussian basis functions for efficient approximation of noisy experimental data. Though the order 8 of MOG works reasonably well in case of our data, it is still a hyper parameter that needs to be tuned for approximating diverse data sets. For first convergence, we have used optimized existing algorithms [19] to compute the parameters of MOG during function estimation.

\subsection{Abel deconvolution integral}

The coefficients of Gaussian Model, obtained in the function estimator stage are used to construct a continuous function $F(y, z)$ of the specific order for the variation of intensity in each row (z). These coefficients of MOG are computed by non-linear least square fitting method. In order to obtain the emission function $f(\rho, z)$, the integral form of inverse Abel transform [10], as given by equation (4), has been used. Thus, the estimated continuous function $F(y, z)$ is Abel deconvoluted, and the data corresponding to emission function in each row are acquired [12, 13]. For deconvolution to be meaningful cylindrical symmetry is mandatory. Though the experimental results are not $100 \%$ symmetric we actually ignore the asymmetry as the variations are minimal. This is also the reason why we do only deconvolution on one side. Assuming cylindrical symmetry the deconvolution process, irrespective of whether it is done on left or the right half, will give the same result. Therefore, in order to address this issue, only one side of 
the projected image $F(y, z)$ is deconvoluted to obtain one side of $f(\rho, z)$ and then it is reflected along the vertical axis (Z) for complete reconstruction of $f(\rho, z)$. In practice, the deviation from symmetry is minimal when compared with the average counts, and hence, it is reasonable to tackle the asymmetry issue in such manner. This procedure is followed for all the rows of the enhanced image to obtain spatial field information of the entire image.

The analytical solution of Abel inversion can be derived as following. For reducing the complexity, we have shown the derivation for one Gaussian basis i.e. $F(y, z)=a e^{-\left(\frac{y-b}{c}\right)^{2}}$ with $a=1, b=0, c=1$. Now, $f(\rho, z)$ can be obtained from $F(y, z)$ by

$$
\begin{aligned}
f(\rho, z) & =\frac{-1}{\pi} \int_{y=\rho}^{\infty} \frac{d F}{d y} \frac{d y}{\sqrt{y^{2}-\rho^{2}}}, \forall z \\
& =\frac{-1}{\pi} \int_{y=\rho}^{\infty} a e^{-\left(\frac{y-b}{c}\right)^{2}}(-2)\left(\frac{y-b}{c}\right)\left(\frac{1}{c}\right) \frac{d y}{\sqrt{y^{2}-\rho^{2}}} \\
& =\frac{2}{\pi} \int_{y=\rho}^{\infty} \frac{y}{\sqrt{y^{2}-\rho^{2}}} e^{-y^{2}} d y, \\
& \text { substituting } \mathrm{a}=1, \mathrm{~b}=0, \mathrm{c}=1 \\
& =\frac{2 e^{-\rho^{2}}}{\pi} \int_{u=0}^{\infty} e^{-u^{2}} d u, \text { substituting } \sqrt{y^{2}-\rho^{2}}=u
\end{aligned}
$$

Gaussian error function is a special integral given by the following equation.

$$
\operatorname{erf}(x)=\int_{t=0}^{x} \frac{2 e^{-t^{2}}}{\sqrt{\pi}} d t
$$

Therefore, $f(\rho, z)$ can be reduced further to

$$
\begin{aligned}
f(\rho, z) & =\frac{e^{-\rho^{2}}}{\sqrt{\pi}} \int_{0}^{\infty} \frac{2 e^{-u^{2}}}{\sqrt{\pi}} d u \\
& =\frac{e^{-\rho^{2}}}{\sqrt{\pi}} \operatorname{erf}(\infty) \\
& =\frac{e^{-\rho^{2}}}{\sqrt{\pi}}, \because \operatorname{erf}(\infty)=1
\end{aligned}
$$

This approach can be extended to higher order Gaussian basis functions in the similar manner.

\subsection{Spatial distribution of data}

One of the important aspects of this processing is to arrange the data in spatial coordinate frame. After performing Abel deconvolution, the deconvoluted image is obtained in pixel coordinate frame which needs to be converted into spatial domain in order to analyse better in real world units such as $\mathrm{cm}, \mathrm{mm}$, etc. In order to achieve this, we have used the stored relevant scaling information to convert from pixel coordinate frame to spatial coordinate frame. The scaling information is obtained by estimating the pixel resolution from images of standard calibration targets. By proper utilization of this information, Abel inverted image is represented in spatial coordinate frame. The pseudo codes for coefficient extraction and Abel deconvolution are given in Algorithm 1 and Algorithm 2 respectively. 


\subsection{Proposed algorithm}

Algorithm 1 : Pseudo Code for Coefficient Extraction

1. input : line-of-sight integrated image

2. processing :

(a) Compute size of the input image. Let row and col be the dimensions of the input image.

(b) Initialize a matrix to store coefficients of approximated curve. Let num represents the number of coefficients of the approximating curve. Then the coefficient matrix could be initialized as a null matrix of size (row, num)

(c) for all the rows of input image

i. Apply Mixture of Gaussian model(MOG) as given in equation (7) to approximate intensity $F(:, z)$ of each row $(z)$ with respect to columns $(y)$.

$$
F(y, z)=\sum_{n=1}^{8}\left(a_{n} \exp ^{-\left(\frac{y-b_{n}}{c_{n}}\right)^{2}}\right), \forall z
$$

where $a_{n}, b_{n}$ and $c_{n}$ are the 24 coefficients to be estimated for each row. Therefore, the value of num is 24 in this MOG model.

ii. Store all the 24 coefficients corresponding to each row for further processing.

(d) end

3. output : As a result of this process, the coefficient matrix of MOG model of the whole input data are obtained as prerequisites for performing Abel deconvolution.
Algorithm 2 : Pseudo Code for Abel Deconvolution

1. input : The coefficient matrix obtained in the previous process are used as input to Abel deconvolution process.

\section{2. processing:}

(a) Create a matrix to store deconvoluted spatial field information.

(b) for all the rows of input image

i. Construct the continuous Mixture of Gaussian(MOG) model as given in equation (7) using the coefficient matrix corresponding to each row.

ii. Construct the smoothed projection $F(y, z)$, and compute the derivative of continuous MOG $F(y, z)$ for each row.

iii. Perform Abel integration using equation (4) to obtain spatial field $f(\rho, z)$ for each row.

$$
f(\rho, z)=\frac{-1}{\pi} \int_{\rho}^{\infty} \frac{d F}{d y} \frac{d y}{\sqrt{y^{2}-\rho^{2}}}
$$

iv. Stack the spatial field data $f(\rho, z)$ corresponding to each row to obtain Abel deconvoluted information of the whole image.

(c) end

3. output : The outcome of this process is the spatial field data which corresponds to the given line-of-sight projected image. In the final process, this spatial field information is transformed from pixel coordinate frame to spatial coordinate using appropriate scaling information. 

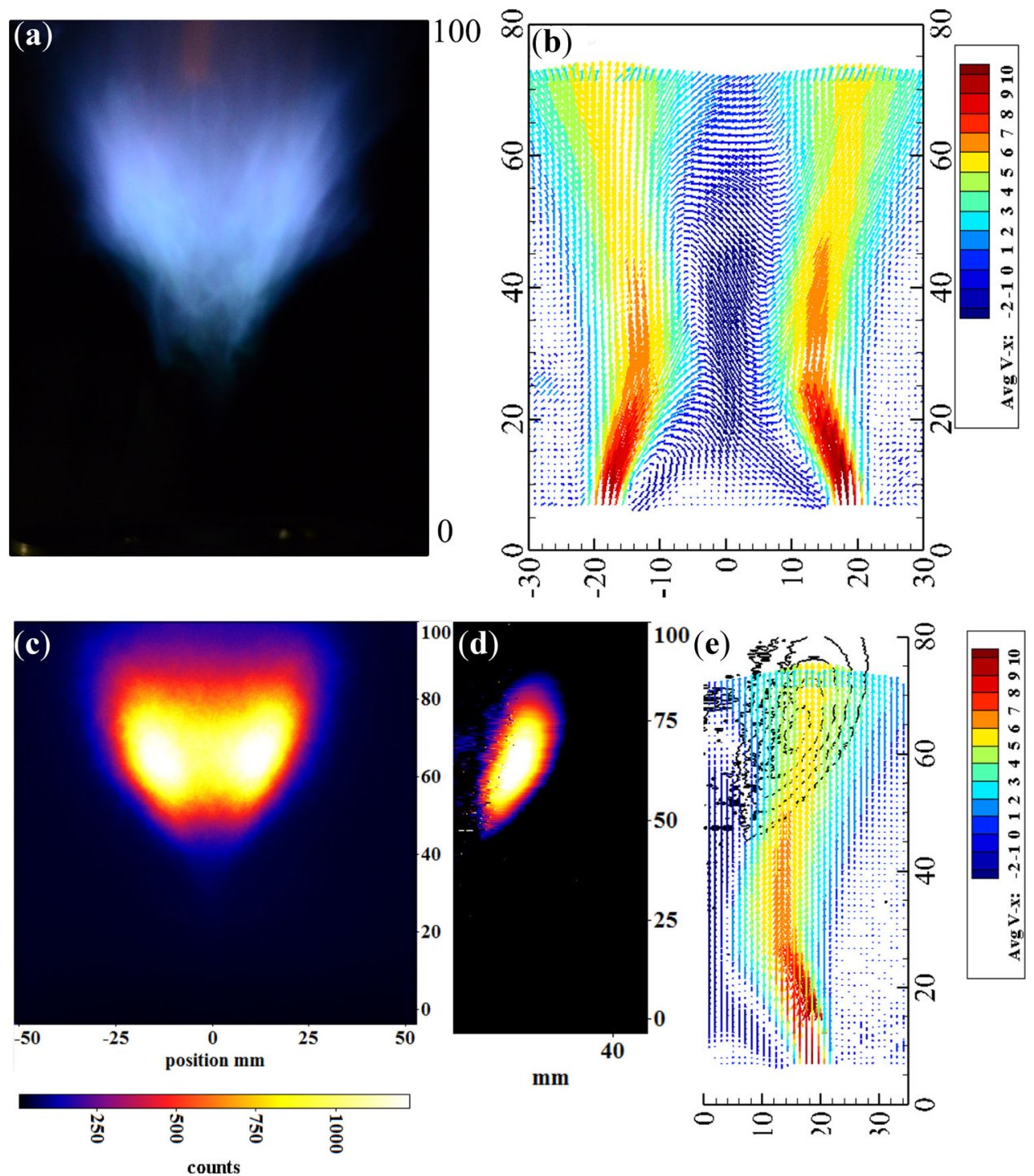

Figure 6. (a) Photograph of flame from a standard swirl burner. (b) Time averaged 2D-velocity distribution along the axial plane. (c) Time averaged $\mathrm{OH}$ chemiluminescence image. (d) Abel deconvoluted image. (e) Multi-layered velocity field overlapped with Abel deconvoluted $\mathrm{OH}^{*}$ image as contour plot.

\subsection{Computational complexity}

One major advantage of using mixture of Gaussian is the number of transcendental operations involved in computing the coefficients of fitted curve. This model requires three transcendental operations for computing $a_{n}, b_{n}, c_{n}$ (Eq. (7)), in contrast with ASAI [13] which requires four transcendental operations between two discrete data points. The worst case time complexity of the proposed algorithm (4.5) is $O(n)$ because it takes $O(1)$ time in computing the coefficients of each $n$ row of an $n \times m$ image where as the time complexity of ASAI is $O\left(n^{2}\right)$ [13]. Though FAI algorithm [13] uses less number of transcendental operations than the proposed algorithm, it doesn't adapt to the changes in characteristics of data points. The proposed algorithm can adapt this change as the coefficients involved in our model depend on input data. Also, it performs less but not the least number of transcendental operations. It has storage space complexity of $O\left(n^{2}\right)$ in order to store the resulting image. The coefficients of the model can be pre-computed and stored prior to the execution of the Abel integration which helps to boost the speed. The Abel integration algorithm is also of $O(n)$ time complexity which can be computed in the similar manner as in coefficient generation. Thus, the overall time complexity of the algorithm is $O(n)$ by the addition rule of measuring worst case time complexity [20]. Above all, the proposed algorithm meets the requirement of using minimal transcendental operations with the ability to capture necessary changes in the input data. 

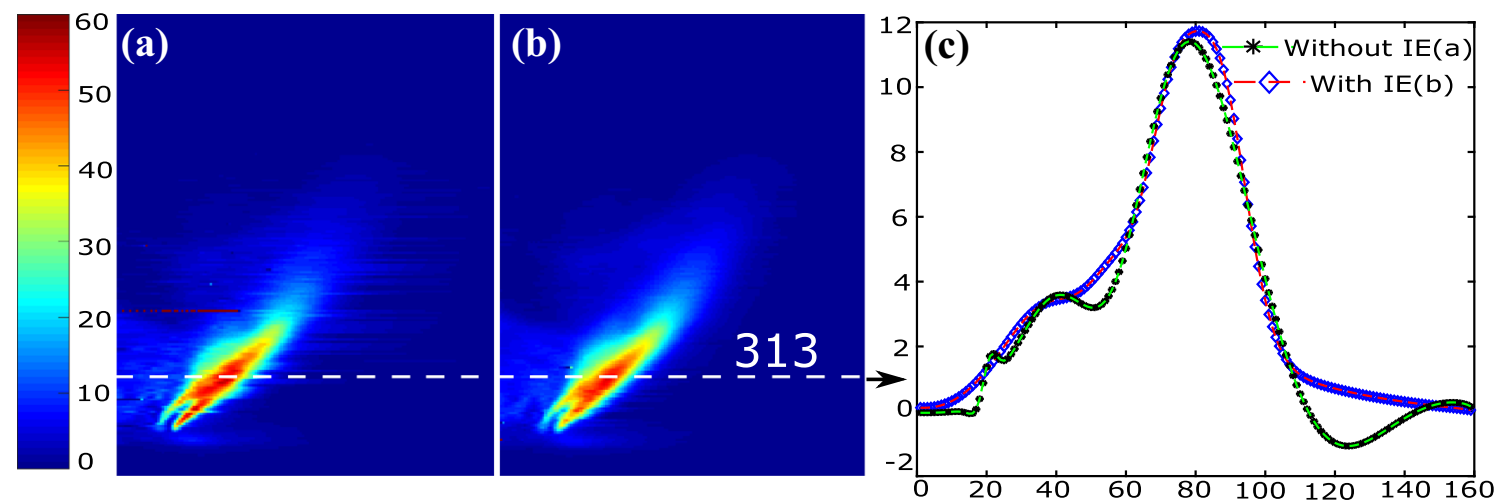

Figure 7. Abel deconvolution without and with image enhancement (IE) are shown in (a) and (b) respectively. It is evident that the edge adaptive smoothing of input image alleviates the adverse effect of unwanted noise signals in the deconvoluted image. (c) Intensity profile along a fixed row (here, 313) of deconvoluted images with and without IE.

\section{Experimental details and analysis}

The intended application of the newly developed algorithm is explained in figure 6 . The exemplary images shown correspond to optical and laser diagnostic investigations conducted in a swirl stabilized, standard burner with methane as fuel [21]. Figure 6(a) shows the photograph of the flame, and the corresponding time averaged $\mathrm{OH}^{*}$ image captured using an ICCD camera (La Vision, Model: Nanostar, 12 bit, $1280 \times 1024$ pixels) is shown in figure 6(c). The burner is operated with an air and fuel flow rates of $200 \mathrm{lpm}$ and $18.9 \mathrm{lpm}$ respectively, resulting in a thermal power of $11.1 \mathrm{~kW}$. The burner operated in nonpremixed mode at atmospheric conditions produced a turbulent flame at the investigated conditions. The $\mathrm{OH}^{*}$ images are captured with an exposure time of 100 microseconds, and 300 such instantaneous images are used to generate the time averaged image. In general the dominant noise source in scientific grade CCD and ICCD cameras are shot noise (the variations in the photoelectrons generated at each pixel) and the dark current read noise. The dark current read noise is negligible in our case owing to the short exposure and readout times. Post processing of the raw images was done to correct the camera chip sensitivity or shot noise and background noise (signals coming from unwanted background light). Shot noise usually follow Poisson distribution. For large numbers, the Poisson distribution tend to follow normal distribution, and the elementary events such as photons, electrons etc. are no longer observed individually. Typically this makes shot noise in actual observations indistinguishable from true Gaussian noise, and hence, the necessity of adaptive smoothing in image enhancement process. Variations in the camera chip sensitivity is normalized using an ensembleaverage image of a uniformly illuminated screen. The ensemble averaged background image is obtained using the

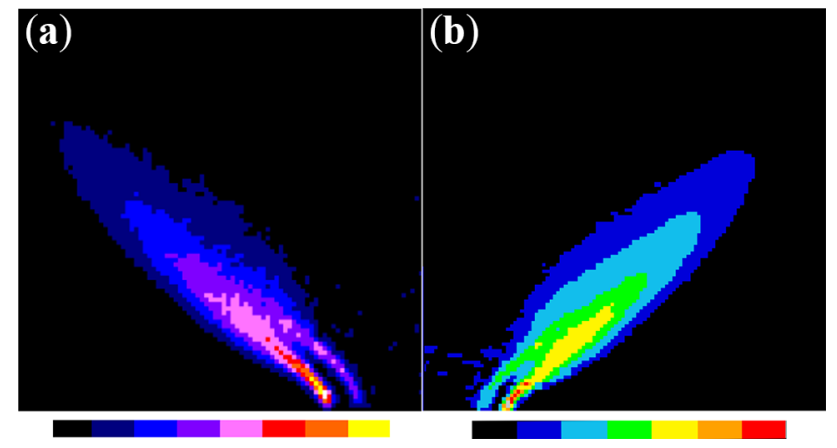

Figure 8. Qualitative comparison of the proposed method (b) with one of the state-of-the-art methods (a) from literature. The exemplary image shows the deconvoluted chemiluminescence image from a swirl stabilized burner. Since any one side is reflected to reconstruct the complete image, one can compare either side of both outcomes to draw inferences regarding the efficacy of the method.

same camera and intensifier settings with the flame extinguished. By uniform illumination in the experimental setup we try to minimize noise due to variable illumination, though not completely, and by edge adaptive smoothing we try to remove the AWGN noise up to some extent. It is evident from the flame photograph that the flame is lifted from the burner exit. One of the goals is to understand the influence of underlying local turbulent velocity field on the flame stabilization and heat release o $\mathrm{r}$ in other words, the underlying physical mechanism that enables the flame to stabilize against such high velocity flows. One way is by pixel-to-pixel comparison of the flame data with the corresponding velocity information. This implies that spatially resolved information about the velocity field along with the information about the flame/reactant species is indispensable.

The 2D vector field is obtained using a laser based diagnostic technique called Particle Image Velocimetry 
(PIV) [22], where the displacement vector is calculated using auto-correlation or cross correlation techniques. Using the time between the laser shots and the physical pixel resolution of the camera the velocity is then calculated from the displacement vector. As the PIV data is spatially resolved in $2 \mathrm{D}$, the missing element in this analysis is the spatially resolved information about the reactive species. So, the Abel deconvolution algorithm is employed on the time averaged $\mathrm{OH}^{*}$ images to obtain the $2 \mathrm{D}$ spatially resolved information as shown in figure 6(d). Due to the axial symmetry only one half of the image is shown. The time averaged and planar vector field corresponding to the flow conditions is shown in figure 6(b). Here the red and black colour vectors indicate the maximum and minimum velocities respectively. As $\mathrm{OH}^{*}$ is a measure of the heat release, by overlapping the Abel deconvoluted $\mathrm{OH}^{*}$ image on the 2D-PIV image a deeper insight into the turbulencechemistry (heat release) interactions can be obtained. Such a multi-layered image is shown in figure 6(e) where the planar velocity field is overlapped with Abel deconvoluted $\mathrm{OH}^{*}$ images in contour plot. The image gives a good impression of the flow field conditions in which the flame is stabilizing. Just ahead of the flame front there is an apparent deceleration of the flow field and also the $\mathrm{OH}^{*}$ contours of maximum heat release are located in regions of relatively lower local velocities. Here the reader is reminded that $2 \mathrm{D}$ images do not give the complete picture as the turbulence flame interactions in such flows are highly three dimensional in nature. However the example clearly highlights the importance and the benefits of the Abel deconvolution algorithm in analysis of such combustion related phenomena.

In order to validate the relevance of image enhancement, we have compared the Abel deconvolution process with and without the proposed pre-processing i.e. edge adaptive Gaussian smoothing in an exemplary deconvoluted flame image as shown in figure 7. The image enhancement process imposes additional degree of smoothness on the input image which is reflected in the deconvoluted images. From this comparison, we report that the edge adaptive smoothing lessens the effect of unwanted signals in such applications. From figure 7(c), it is apparent that the MOG model still lacks smooth transition in intensity, mainly due to high fluctuation in raw data as a result of unconstrained noisy environment. However, enhancing the degree of smoothness prior to fitting MOG model resolves this issue up to some extent, as can be inferred from figure 7(b). Further, we have compared our approach with one of the state-of-the-art methods [23] dealing with similar issues as ours. As figure 8 indicates, the state-of-the-art method suffers from adverse effects of noisy environment, however, the proposed algorithm could suppress these unwanted signals and hence, it could impose higher degree of smoothness by simultaneously leveraging the image enhancement and Gaussian mixture model. The variation of intensity towards the axis of symmetry is expected due to the influence of heat release from the burner exit. The stateof-the-art method, as shown in figure 8(a), does not have the provision to regularize inversion by imposing smoothness constraint on acquired raw data. This results in abrupt fluctuations in the reconstructed scalar field. However, the proposed reconstruction, as shown in figure 8(b), alleviates this effect through constrained regularization. Due to unavailability of continuous reconstructed profile in the state-of-the-art method, we have quantized our continuous reconstructed profile and compared with the available quantized version of the state-of-the-art method. The efficacy of inversion can be suitably inferred from this comparison, as shown in figure 8 , by focusing on each quantized region of both the images. The proposed method has fewer fluctuations in each quantized region of the state-of-the-art method that leads to marginally superior inversion. However, as the final reconstruction is greatly influenced by the quality of the raw data, which again depends on the experimental settings, it is not possible to have a concrete conclusion on the superiority of the new method from the images here. What is clear from figure 9 is that the proposed MOG based Abel inversion through constrained regularization is proved to be equally good from the application of point of view and hence, can be suitably used as an alternative to the conventional methods.

The obtained results asserts that the proposed technique using standard Abel inversion for the application in combustion research is useful where limited infrastructure is available for acquiring the expensive laser diagnostics methods like planar laser induced fluorescence of $\mathrm{CH}$ radical (CH-PLIF) image. Our findings indicates, the application of MOG prior to deconvolution approximates the discrete data points relatively better than the other compared methods. Based on the intensity profile and qualitative comparison among various standard methods, we believe that the proposed framework is an alternative way to achieve the desired results and it works satisfactorily well compared to other standard techniques in the adopted experimental setting.

\section{Conclusion}

Abel transformation finds its applications in many research fields including plasma diagnostics, X-ray radiography etc. In this paper, we critically analyze the inherent issues of Abel deconvolution and propose a generic framework to tackle these issues up to some extent. The proposed algorithm gave maximum efficacy with the adopted image acquisition settings. Moreover, the proposed framework offers a provision to eliminate a certain amount of unwanted signals which is essential to be removed in many scenarios. Of particular interest is the extraction of $2 \mathrm{D}$ and 
spatially resolved electronically excited emission from the hydroxyl radical $\left(\mathrm{OH}^{*}\right.$ chemiluminescence) in axisymmetric flames. The deconvoluted $\mathrm{OH}^{*}$ image overlapped with the time-averaged 2D velocity plot from the same burner highlights the importance and effectiveness of the algorithm in combustion research. Above all, the algorithm meets the requirement of performing Abel inversion in $O(n)$ time and $O\left(n^{2}\right)$ space complexity through regularization by imposing smoothness constraint over acquired raw images.

\section{References}

[1] Trupti K 2011 Reaction kinetics modeling of $\mathrm{OH}^{*}, \mathrm{CH}^{*}$, and $C 2 *$ chemiluminescence. Ph.D. thesis

[2] Najm H N, Paul P H, Mueller C J and Wyckoff P S 1998 On the adequacy of certain experimental observables as measurements of flame burning rate. Combust. Flame 113(3): 312-332

[3] Ikeda Y, Kojima J and Nakajima T 2005 Basic aspects of oh(a), ch(a) and c2(d) chemiluminescence in the reaction zone of laminar methane-air premixed flames. Combust. Flame 140(1): 34-45

[4] Boehly T R 1996 Abel inversion of emission and backlighting images. LLE Rev. 66: 66-72

[5] Dasch C J 1992 One-dimensional tomography: a comparison of abel, onion-peeling, and filtered backprojection methods. Appl. Opt. 31(8): 1146-1152

[6] Tikhonov A N 1975 Inverse problems in heat conduction. $J$. Eng. Phys. Thermophys. 29(1): 816-820

[7] Simons S N and Yuan Z-G 2003 The filtered abel transform and its application in combustion diagnostics. 1995 Fall Meeting, Western States Section, sponsored by The Combustion Institute, Stanford, California, October 30-31, 1995

[8] Daun Kyle J, Thomson Kevin A, Liu Fengshan and Smallwood Greg J 2006 Deconvolution of axisymmetric flame properties using tikhonov regularization. Appl. Opt. 45(19): $4638-4646$
[9] Sizikov V S, Evseev V, Fateev A and Clausen S 2016 Direct and inverse problems of infrared tomography. Appl. Opt. 55(1): 208-220

[10] Abel N H 1827 Recherches sur les fonctions elliptiques. $J$. fur die reine und angewandte Math. 2: 101-181

[11] Deutsch M and Beniaminy I 1982 Derivative-free inversion of abel's integral equation. Appl. Phys. Lett. 41(1): 27-28

[12] Deutsch M and Beniaminy I 1983 Inversion of abel's integral equation for experimental data. J. Appl. Phys. 54(1): 137-143

[13] Gueron S and Deutsch S 1994 A fast abel inversion algorithm. J. Appl. Phys. 75(9): 4313-4318

[14] Petrou M and Petrou C 2010 Image processing: the fundamentals. Hoboken: Wiley

[15] De Moor B 1994 Total least squares for affinely structured matrices and the noisy realization problem. IEEE Trans. Signal Process., 42(11): 3104-3113

[16] Press William H, Flannery Brian P, Teukolsky Saul A and Vetterling William T 1989 Numerical recipes, volume 3. Cambridge University Press, Cambridge

[17] Dyn N 1989 Interpolation and approximation by radial and related functions. Approximation Theory VI, 1(s 1): 211-234

[18] Ross S M 2014 Introduction to probability and statistics for engineers and scientist. India: Academic Press, New Delhi, 5 edition

[19] MATLAB Optimization Toolbox 2014b The mathworks inc. Natick, MA

[20] Allen W M 1996 Algorithms, data structures, and problem solving with $\mathrm{C}++$. Boston: Addison Wesley Longman Publishing Co., Inc

[21] Jarpala R, Burle N V S A, Voleti M and Sadanandan R 2017 Effect of swirl on the flame dynamics and pollutant emissions in an ultra-lean non-premixed model gt burner. Combust. Sci. Technol. 189(10): 1832-1848

[22] Willert C E and Gharib M 1991 Digital particle image velocimetry. Exp. Fluids 10(4): 181-193

[23] Sadanandan R, Kutne P, Steinberg A and Meier W 2012 Investigation of the syngas flame characteristics at elevated pressures using optical and laser diagnostic methods. Flow Turbul. Combust. 89(2): 275-294 